\title{
CONDIÇÕES DE SAÚDE DOS IDOSOS EM CORRESIDÊNCIA
}

\section{HEALTH CONDITIONS OF THE ELDERLY IN CORRESIDENCE}

\author{
Caroligia Pereira de Souza ${ }^{1}$, Sandiele Patez Rocha ${ }^{1}$, Alba Benemérita Alves Vilela ${ }^{2}$, Talita \\ Santos Oliveira Sampaio ${ }^{1}$, Lucas Silveira Sampaio ${ }^{1}$ \\ Faculdade Independente do Nordeste ${ }^{1}$, Universidade Estadual do Sudoeste da Bahia ${ }^{2}$
}

\section{Abstract}

The objective of this research is to evaluate the health conditions of elder people who live in coresidence. It is a cross-sectional, descriptive study with a quantitative approach. It was carried out in the Family Health Units in the municipality of Vitória da Conquista, Bahia, Brazil, with 239 elderly people living in corresidences in the urban area. For data collection, we used interviews, through a semi-structured questionnaire, the adapted Brazil Old Age Schedule Questionnaire and the Health Status Questionnaire. The tabulation was performed using Excel 2015 software and the data were analyzed by Statistical Package the for Social Sciences for Windows. Through the data obtained in this study, a prevalence of females (77\%) was observed. Most corresident elderly people live with their grandchildren (57.3\%), followed by their sons (55.2\%) and daughters (54\%). By analyzing the health conditions of the elderly, we observed that (53.8\%) of the elderly considered their health only as Regular, with the main pathologies referred to being Hypertension (80.5\%), Rheumatological diseases (35.1\%) and Diabetes (26.8\%). We concluded that the health condition of the elderly in corresidence, was marked by the prevalence of chronic diseases such as hypertension, rheumatological diseases and diabetes, which can directly reflect on the self-perception of health since the majority of the elderly reported considering having a regular health condition. The study contributes to the reflection of health professionals in relation to the provision of health care as well as to the relatives of the elderly, to promote a better quality of life for this population within the context of public health.

Keywords: Elderly. Health of the elderly. Family. Health conditions.

\section{Resumo}

O objetivo da presente pesquisa é avaliar as condições de saúde dos idosos que vivem em corresidência. Trata-se de um estudo com delineamento transversal, do tipo descritivo $e$ abordagem quantitativa. Foi realizado nas Unidades de Saúde da Família do município de Vitória da Conquista - Bahia, com 239 idosos em corresidência residentes na zona urbana. Para a coleta de dados foi utilizado a entrevista, através de questionário semiestruturado, o questionário Brazil Old Age Schedule adaptado e o Questionário de Estado de Saúde. A tabulação foi realizada através do software Excel 2015 e os dados foram analisados no Statistical Package for The Social Sciences para Windows. Através dos dados obtidos neste estudo observou uma prevalência do sexo feminino (77\%). Os idosos corresidentes vivem em sua maioria com netos (57,3\%), seguido dos filhos $(55,2 \%)$ e filhas (54\%). Ao analisar as condições de saúde dos idosos, foi observado que $(53,8 \%)$ dos idosos consideravam sua saúde apenas Regular, sendo que as principais patologias referidas foram Hipertensão (80,5\%), doenças Reumatológicas (35,1\%) e Diabetes (26,8\%). Conclui-se que a condição de saúde dos idosos em corresidência foi marcada pela prevalência de doenças crônicas como hipertensão, doenças reumatológicas e o diabetes, o que pode refletir diretamente na autopercepção de saúde onde a maioria dos idosos relataram considerar ter uma condição de saúde regular. $O$ estudo contribui com a reflexão dos profissionais de saúde em relação à prestação de assistência de saúde como também aos familiares dos idosos, para a promoção de uma melhor qualidade de vida para essa população dentro do contexto de saúde pública.
Palavras-chave: Idoso. Saúde do idoso. Família. Condições de Saúde. 
Introdução

Um país é reconhecido como estruturalmente envelhecido quando no mínimo $7 \%$ de sua população é composta por indivíduos de 60 anos ou mais, o que demonstra que a população brasileira está envelhecendo, já que o Brasil possui mais de 28 milhões de pessoas idosas, que representam $13 \%$ da população do país. Diante disso, estima-se que em 2025 o Brasil terá uma população de 35 milhões de idosos, sendo o sexto maior país do mundo em número de pessoas idosas. ${ }^{1-2}$

Segundo Meira $^{3}$, esse envelhecimento populacional representa um desafio. A rapidez do crescimento da expectativa de vida gera um aumento das preocupações de gestores, sociedade e da família em atender as demandas dos idosos, principalmente em relação às ações direcionadas ao suporte social. A atenção às pessoas idosas deve ser voltada para as várias proporções de sua vida, tendo como consideração os aspectos de saúde, família, economia e social desses indivíduos. Em relação aos aspectos de família, observa-se que em decorrência do envelhecimento a estrutura familiar está em progressiva transformação, provocando a formação de novos arranjos familiares. Dentre estes, pode-se destacar a corresidência, que é o convívio de pelo menos um idoso com filhos e/ou netos no mesmo ambiente domiciliar. ${ }^{4-5}$

Um fator importante para a ocorrência da corresidência é que a família tem um papel de grande relevância, por ser o contexto social mais próximo do idoso. A família é considerada um ambiente de cuidados de saúde, principalmente quando este idoso é dependente funcionalmente para realização de atividades do dia a dia. Tornando-se a família do idoso uma importante colaboradora na formação de um envelhecimento saudável. ${ }^{6}$

Outro fator relacionado ao crescimento do arranjo da corresidência segundo o estudo de Neri e Rabelo ${ }^{4}$ são as dificuldades financeiras dos filhos e/ou netos, devido às instabilidades no mercado de trabalho principalmente para os mais jovens, e a presença de divórcios que fazem com que filhos retornem a casa dos pais. Diante desse contexto, os idosos se tornam provedores da família, oferecendo suporte emocional, financeiro e de cuidados com os netos para que os filhos possam trabalhar. ${ }^{7-8}$

A corresidência geralmente é benéfica para os idosos e seus familiares, sendo um espaço de compartilhamentos e trocas. Pode refletir positivamente no apoio afetivo e emocional, além de oferecer auxílio instrumental e material. Entretanto, esse convívio intergeracional com filhos e netos pode gerar aspectos negativos na saúde dos idosos principalmente quando ocorre escassez de recursos materiais e há conflitos de interesses. Estudos analisaram que a corresidência não assegura que o idoso irá obter todo auxilio que necessita ou espera em relação a sua saúde. 0 convívio marcado pela presença de diferentes gerações de vida pode favorecer a divergências de opiniões, além de muitos idosos serem resistentes a certas mudanças, o que poderia provocar conflitos no ambiente familiar. Pode-se afirmar que as instabilidades financeiras dos mais jovens podem provocar preocupações, ansiedades e depressão entre os idosos. ${ }^{3-4-8}$

Por essas razões, surge a importância do aprofundamento na temática da corresidência entre idosos e seus familiares, pois este tipo de arranjo pode afetar diretamente no bem estar e na condição de saúde do idoso. Este estudo tem como finalidade contribuir para o desenvolvimento da assistência e no planejamento de novas estratégias de saúde pública, mobilizando assim, mais pesquisas sobre os impactos positivos e negativos que a corresidência pode oferecer para a saúde dos idosos. Além disso, busca ampliar o debate sobre a conformação da família intergeracional ligada a corresidência no contexto do envelhecimento populacional. Com isso, este estudo tem como objetivo avaliar as condições de saúde dos idosos que vivem em corresidência.

\section{Metodologia}

Trata-se de um estudo do tipo descritivo com delineamento transversal e abordagem quantitativa. Este estudo é um subprojeto do projeto intitulado "Arranjo Familiar de Idosos Residentes em Municípios do Nordeste e Sudeste do Brasil", o qual foi realizado por meio de uma proposta de pesquisa em consórcio estabelecido entre o Programa de Pós - Graduação em Enfermagem e Saúde da Universidade Estadual do Sudoeste da Bahia e um grupo de mestrandos. Este estudo está vinculado ao Núcleo de Pesquisa em Saúde do Idoso- NUPESI da Faculdade Independente do NordesteFAINOR.

O estudo foi desenvolvido em duas Unidades de Saúde da Família (USF) do município de Vitória da Conquista - BA. Segundo o Cadastro Nacional de Estabelecimentos (CNS), o município possui 43 USF, porém, a coleta de dados foi realizada em apenas duas USF as quais foram 
selecionadas através de um sorteio simples. Foram incluídos no estudo os indivíduos com idade igual ou superior a 60 anos que viviam em situação de corresidência residentes na zona urbana do município, e que através da aplicação do Mini Exame do Estado Mental (MEEM) mostraram condições cognitivas que permitiram responder aos questionamentos.

Conceitua-se neste estudo o idoso em estado de corresidência como aquele que convive e reside com sua família intergeracional (uma ou mais gerações, ou seja, filhos e/ou netos e/ou bisnetos dentre outros) no mesmo ambiente domiciliar. ${ }^{5}$ Foram excluídos da amostra idosos que apresentaram déficit cognitivo através da aplicação do MEEM, aqueles que recusaram a participar do estudo ou que não foram encontrados em sua residência após três tentativas em dias e horários alternados. Após seguir os critérios de inclusão e exclusão estabelecidos pela pesquisa, a população de estudo totalizou-se em 239 idosos.

O MEEM inclui 11 itens, que demandam respostas verbais a questões de orientação temporal e espacial, atenção, leitura, memória, cálculo, nomeação, seguir comandos verbais e copiar um desenho (polígonos). Para avaliação dos resultados obtidos através do MEEM, foram adotados os seguintes pontos de corte: 19 pontos para idosos analfabetos; 23 pontos para idosos com 1 a 3 anos de escolaridade; 24 pontos para idosos com 4 a 7 anos de escolaridade e 28 pontos para idosos com escolaridade acima de 7 $\operatorname{anos}^{10}$. Escores abaixo destas pontuações indicam um risco de déficit cognitivo requerendo encaminhamento para avaliação neuropsicológica específica.

A coleta de dados foi realizada em duas etapas: Na primeira, foi identificado o número de idosos cadastrados nas USF através da consulta das fichas de cadastramento familiar (ficha A), e com o auxílio dos agentes comunitários de saúde foi feito o levantamento dos idosos que viviam sós e aqueles que corresidiam com os seus familiares. Na segunda etapa foi realizada a entrevista nos domicílios dos idosos selecionados, através dos seguintes instrumentos: primeiro foi aplicado o Mini Exame do Estado Mental (MEEM) para rastreio do déficit cognitivo, após foi aplicado o Questionário semiestruturado obtendo dados de identificação dos participantes, como idade, sexo e composição do arranjo familiar, o questionário Brazil Old Age Schedule (BOAS) adaptado e o questionário de Estado de Saúde. Todos os questionários foram aplicados de forma impressa pelo entrevistador.
O Questionário Brazil Old Age Schedule BOAS é um instrumento de pesquisa que verifica fatores multidimensionais do envelhecimento baseado em outros instrumentos com padrões aceitáveis de validade e confiabilidade. O BOAS foi idealizado a partir de 8 grandes categorias (Informações gerais, saúde física, utilização de serviços médicos e dentários, atividades de vida diária, recursos sociais, recursos econômicos, saúde mental, necessidades e problemas que afetam o entrevistado). ${ }^{11} \mathrm{~A}$ equipe que aplicou os instrumentos foi composta por 6 discentes do curso de Fisioterapia da Faculdade Independente do Nordeste- FAINOR, e a coleta de dados ocorreu 5 vezes por semana no período entre os meses de fevereiro a abril do ano de 2018. Os dados obtidos pelo BOAS e pela Escala de Estado de Saúde foram tabulados e analisados utilizando o programa Statistical Package for The Social Sciences para Windows (SPSS 21.0, 2013, SPSS, Inc, Chicago, IL). Foram calculadas médias, frequências e medidas de dispersão das variáveis.

A pesquisa principal intitulada "Arranjo Familiar de Idosos em Corresidência Residentes em Municípios do Nordeste e Sudeste do Brasil", foi aprovada pelo Comitê de Ética em Pesquisa da Universidade Estadual do Sudoeste da Bahia (CEP-UESB), cujo parecer $n$ ㅇ 102.641, foi registrado observando a Resolução do Conselho Nacional de Saúde (CNS) no 196/96, vigente na época. Sendo assim, foi encaminhado ao CEPUESB uma solicitação de inclusão deste estudo ao projeto principal de acordo a Resolução no 466 de 12 de dezembro de 2012.

A elaboração dos dados foi feita após a emissão do parecer favorável do CEP-UESB de inclusão do presente campo de estudo e da autorização da Secretaria Municipal de Saúde (SMS) de Vitória da Conquista - BA para a realização da pesquisa nas USF. A participação dos idosos foi voluntária, sendo formalizada a aceitação mediante a assinatura do Termo de Consentimento Livre e Esclarecido - TCLE, que foi assinada em duas vias, sendo uma de domínio do informante e a outra da guarda da pesquisadora responsável pelo estudo.

\section{Resultados}

Significados de resolubilidade para os usuários da Dentre a população entrevistada após análise de critérios de inclusão e exclusão, totalizou-se 239 idosos estudados. A média de idade encontrada foi de $72,2 \pm 8,08$, sendo a idade mínima encontrada 60 anos e a máxima 95 anos. Verificou-se uma maior predominância do sexo 
feminino (77\%) e do estado civil casado (42,6\%), sendo relevante também a presença de viúvos $(41,8 \%)$. No que diz respeito ao arranjo familiar da corresidência, identificou-se maior convivência com netos (57,3\%), seguido de filhos (a) $(55,2 \%$ do sexo masculino e $54,0 \%$ do sexo feminino), conforme dados da Tabela 1.

Tabela 1 - Características sociodemograficas e composição do arranjo familiar dos idosos participantes. Vitória da Conquista - BA, 2018.

\begin{tabular}{|c|c|c|c|}
\hline Variáveis & $\%$ resposta & $\mathbf{N}$ & $\%$ \\
\hline Sexo & 100 & & \\
\hline Feminino & & 184 & 77,0 \\
\hline Masculino & & 55 & 23,0 \\
\hline Grau de escolaridade & 97,5 & & \\
\hline Analfabeto & & 81 & 34,8 \\
\hline Primário incompleto & & 108 & 46,4 \\
\hline Primário completo & & 28 & 12,0 \\
\hline $1^{\circ}$ grau completo & & 5 & 2,1 \\
\hline $2^{\circ}$ grau completo & & 10 & 4,3 \\
\hline Superior completo & & 1 & 0,4 \\
\hline Estado conjugal & 99,2 & & \\
\hline Nunca casou & & 4 & 1,6 \\
\hline Casado(a)/morando junto & & 101 & 42,6 \\
\hline Viúvo (a) & & 99 & 41,8 \\
\hline Divorciado(a)/separado & & 33 & 13,9 \\
\hline Composição do arranjo* familiar & 100 & & \\
\hline Esposo(a)/companheiro(a) & & 97 & 40,6 \\
\hline Pais & & 5 & 2,1 \\
\hline Filhos & & 132 & 55,2 \\
\hline Filhas & & 129 & 54,0 \\
\hline Irmãos/irmãs & & 6 & 2,5 \\
\hline Netos(as) & & 137 & 57,3 \\
\hline Outros parentes & & 16 & 6,7 \\
\hline Outras pessoas & & 13 & 5,4 \\
\hline
\end{tabular}

*Cada indivíduo neste quesito pode ter mais de uma resposta

Ao analisar as condições de saúde dos idosos, na Tabela 2 foi possível observar que $53,8 \%$ dos idosos consideravam sua saúde apenas regular, sendo que as principais patologias referidas foram Hipertensão (80,5\%), Doenças Reumatológicas $(35,1 \%)$ e Diabetes $(26,8 \%)$. 
Tabela 2 - Composição da autopercepção de saúde das pessoas entrevistadas. Vitória da Conquista BA, 2018.

\begin{tabular}{|c|c|c|c|}
\hline Variáveis & $\%$ de resposta & $\mathbf{n}$ & $\%$ \\
\hline Autopercepção da saúde & 98,7 & & \\
\hline Excelente & & 3 & 1,3 \\
\hline Muito boa & & 10 & 4,2 \\
\hline Boa & & 65 & 27,5 \\
\hline Regular & & 127 & 53,8 \\
\hline Má & & 31 & 13,1 \\
\hline Doenças/acidentes autoreferidas & 84,1 & & \\
\hline Hipertensão arterial sistêmica & & 190 & 80,5 \\
\hline Queda nos últimos 12 meses & & 88 & 38,4 \\
\hline Artrite, reumatismo, artrose & & 81 & 35,1 \\
\hline Diabetes Mellitus & & 63 & 26,8 \\
\hline Incontinência urinária & & 58 & 25,0 \\
\hline Problema de nervoso ou psiquiátrico & & 28 & 12,0 \\
\hline
\end{tabular}

*Cada indivíduo neste quesito pode ter mais de uma resposta

\section{Discussão}

Foi observado neste estudo que a maioria dos idosos em corresidência são do sexo feminino, representando (77\%) dos entrevistados. Resultados semelhantes foram encontrados nos estudos de Meira ${ }^{11}$ onde houve predominância do gênero feminino (79\%). Estudos analisaram que as mulheres possuem uma maior sobrevida devido à busca pelos serviços de saúde em comparação aos homens, e com isso, as ações das políticas são amplamente voltadas para o público feminino oferecendo uma maior assistência de saúde. ${ }^{11-6}$

No que se refere ao arranjo familiar observou-se que a maioria dos idosos relataram morar com netas ou netos $(57,3 \%)$, seguido por filhos $(55,2 \%)$ e filhas $(54,0 \%)$. Já no estudo de Junior et al $^{12}$ a maior parte dos idosos entrevistados (52,4\%) afirmaram morar com cônjuge, e (39,8\%) informaram morar com netos(as). Um estudo de Meira et $\mathrm{al}^{3}$ realizado com idosos corresidentes em Minas Gerais, mostrou que esse arranjo familiar na maioria das vezes composto por netos é explicado pelo fato de os idosos servirem como único apoio para seus familiares. Pois, os filhos saem para trabalhar e cabe aos avós os cuidados com os netos.

Além disso, a dificuldade dos filhos entrarem no mercado de trabalho ou casos de divórcios e separações contribui para a formação da corresidência, uma vez que os idosos podem oferecer moradia e dinheiro. Famílias brasileiras que possuem idosos como membros estão em melhores condições financeiras, do que aquelas que não possuem membros idosos. ${ }^{13-4}$

A família é formada como grupo de pessoas unidas por meio de laços e/ou grau de parentesco e que vivem no mesmo ambiente, permitindo as relações de cuidados, atenção, intimidade e apoio, sendo elementos essenciais para um meio harmônico durante o curso da vida. Dessa forma, o convívio familiar tem um papel primordial no processo de envelhecimento. Para os idosos a corresidência acontece principalmente devido ao conforto estabelecido pela presença de pessoas próximas, o que acarreta em bem-estar biopsicossocial, satisfação, sentimento de amor e apoio. ${ }^{1}$

Com relação à autopercepção de saúde, a maioria dos idosos em situação de corresidência $(53,8 \%)$ consideram ter uma saúde "Regular" e $(27,5 \%)$ relatam ter uma condição "Boa" de saúde. O estudo de Silva, Junior e Vilela $^{6}$ ao analisar a autopercepção de saúde dos idosos, foi evidenciado que a maioria $(59,1 \%)$ relataram uma condição negativa de saúde. A corresidência pode ser um modo de vivência vantajosa, confirmando que a família para o idoso é uma importante aliada na construção de um envelhecimento saudável.

Meira et al. $^{3}$ observaram que $(60,6 \%)$ dos idosos relataram considerar seu estado de 
saúde "Bom". Resultados diferentes em relação à autopercepção de saúde podem estar relacionado ao fato de o envelhecimento conduzir diferentes atitudes diante às limitações e incapacidades, podendo levar em alguns casos à adaptações diante das mudanças. Outra possibilidade está na maior aceitação dessas modificações do estado de saúde, uma vez que essas alterações fazem parte da senescência. Sendo assim, os idosos relacionam a perda da capacidade funcional ao envelhecimento e não à perda da saúde, com isso torna-se comum afirmarem possuírem boa saúde para a idade quando são entrevistados. ${ }^{3}$

A autopercepção de saúde se baseia em critérios subjetivos, levando em consideração a percepção de fatores biológicos, psicológicos e sociais de cada idoso em sua individualidade. Além disso, quando se trata de saúde no envelhecimento, atribui não só à ausência de doenças, mas também um equilíbrio entre fatores multidimensionais, como idade, sexo, escolaridade, estado civil, apoio familiar, condições de saúde, estilo de vida, entre outros. . $^{6-}$ 14

A corresidência entre familiares e idosos, tem uma importante relevância por ser o contexto social mais próximo do idoso e também porque os relacionamentos preservados por corresidentes em seus arranjos domiciliares têm implicações positivas para a saúde, já que oferece apoio ao idoso. Entretanto, a presença de conflitos entre os idosos e a família, comumente por divergências de ideias entre gerações, podem acontecer com frequência. Considera-se que o fato do idoso ser, em geral, o provedor emocional e financeiro, além de assumir o papel de cuidar dos netos pode gerar acúmulo de atividades limitando sua privacidade e tranquilidade, e com isso causando estresse e preocupação, esses fatores podem tornar a corresidência negativa e afetar diretamente na condição de saúde da pessoa idosa. ${ }^{6-8}$

Quanto à presença de patologias nos idosos entrevistados, o atual estudo revelou um perfil de morbidade. Grande parte dos idosos em corresidência possuem Hipertensão Arterial Sistêmica (HAS) $(80,5 \%)$, seguido de doenças reumatológicas $(35,1 \%)$ e diabetes mellitus $(26,8 \%)$. Esses resultados foram semelhantes aos do estudo de Junior et al. ${ }^{12}$ onde mostrou que $(70,7 \%)$ possuem HAS, destacando ainda as doenças reumatológicas e diabetes como doenças crônicas que mais afetam os idosos em estado de corresidência.

Da mesma maneira que ocorreu a transição demográfica, aconteceu também a transição epidemiológica, onde se observa que o atual padrão de doença é composto principalmente por patologias classificadas como crônicas não transmissíveis. ${ }^{6}$

A hipertensão e diabetes são os tipos de doenças crônicas mais presente nos idosos e costumam ser um grande obstáculo para os sistemas de saúde no mundo todo. No Brasil, pressupõe-se que em 2030 haverá 11,3 milhões de pessoas diagnosticadas com estas doenças. Estudos também confirmam que as pessoas diagnosticadas com doenças crônicas precisam de mais recursos para serem acompanhadas, além de outros custos associados à dor, ansiedade e menor qualidade de vida dos pacientes e familiares. Apesar disso, as doenças crônicas estão fortemente relacionadas à autopercepção de saúde, podendo fazer com que os idosos percebam de forma negativa a própria saúde, o que justifica considerar sua própria saúde como "Regular". ${ }^{6-7}$

Rabelo e $\mathrm{Neri}^{4}$ explicam que as condições de saúde física podem interferir na manutenção de papéis e funções do idoso dentro da família, já que dependem do nível de desempenho nas atividades básicas e instrumentais da vida diária e da presença de doenças crônicas, a qual a incidência aumenta com a idade. A existência de doenças está vinculada com a redução do envolvimento em atividades sociais complexas que exigem independência física, dessa forma, a redução da participação em atividades sociais pode afetar diretamente na autopercepção de saúde desses idosos. $^{14}$

É importante destacar que a presença de doenças crônicas pode contribuir para a formação de novos arranjos familiares, como o convívio intergeracional, uma vez que idosos com doenças necessitam mais do suporte de seus familiares, pois entende-se que o idoso necessita de cuidados médicos e / ou da família (cônjuges, filhos e/ou netos) para melhor qualidade de vida, além da necessidade, na maioria das vezes, de auxílio nas atividades do dia-a-dia. Para muitos desses idosos, a vantagem da corresidência está relacionada à companhia, ao suporte emocional, amparo financeiro e cuidados físicos, tanto por parte dos pais quanto dos filhos, como também ajudar a administrar os custos para sobrevivência, o que não seria possível fora do ambiente familiar. ${ }^{14,11}$

\section{Considerações finais}

A condição de saúde dos idosos em corresidência, no presente estudo, foi marcada 
pela prevalência de doenças crônicas, como hipertensão arterial, doenças reumatológicas e diabetes mellitus, o que pode refletir diretamente na autopercepção de saúde onde a maioria dos idosos relataram considerar ter uma saúde "Regular". Destacando também que a presença de doenças crônicas pode contribuir para a formação de novos arranjos familiares, como o convívio intergeracional, uma vez que idosos com doenças crônicas necessitam mais do suporte de seus familiares. Além disso, foi possível observar que condições de saúde física podem interferir na manutenção de papéis e funções sociais, já que a existência de doenças está vinculada com a redução do envolvimento em atividades sociais complexas que exigem independência física. Com isso, entender as condições de saúde dos idosos em corresidência é de extrema importância para que haja aprofundamento, já que este tipo de arranjo familiar pode afetar diretamente no bem estar e na condição de saúde do idoso.

Este estudo tem como finalidade contribuir para o desenvolvimento da assistência e no planejamento de novas estratégias de saúde pública, mobilizando assim, mais pesquisas sobre os impactos positivos e negativos que a corresidência pode oferecer para a saúde dos idosos. Além disso, o presente estudo busca ampliar o debate sobre a coabitação intergeracional no contexto do envelhecimento populacional. Dessa forma, é importante a realização de novos estudos com maior aprofundamento da temática, utilizando outras metodologias com maiores amostras e/ou população e em outras regiões do país para assim surgir novas discussões e estimular maior exploração sobre o assunto envelhecimento e suas especificidades que abrangem a condição de saúde do idoso no convívio intergeracional.

\section{Referências}

1. Araújo LF, Castro JLC, Santos JVO. A família e sua relação com o idoso: Um estudo de representações sociais. Psicol. Pesqui. 2018; 12(2): 14-23

2. Instituto Brasileiro de Geografia e Estatística (IBGE). (2018). Projeção da população 2018: o número de habitantes do país deve parar de crescer em 2047. Brasília: Estatísticas Sociais. Recuperado em 10 de novembro de 2020, de http://agenciadenoticias.ibge.gov.br/agenciasala-de-imprensa/2013-agencia-denoticias/releases/21837-projecao-da-populacao2018-numero- de-habitantes-do-pais-deve-pararde-crescer-em-2047
3. Meira SS, Vilela ABA, Casotti CA, Anjos ACF, Silva DM. Considerações acerca das condições de vida de idosos no Vale do Jequitinhonha, Minas Gerais - Brasil. SERV. SOC. REV. 2014; 17(1): 159-177.

4. Rabelo DF, Neri AL. Tipos de configuração familiar e condições de saúde física e psicológica em idosos. Cad. Saúde Pública. 2015; 31(4): 874-884.

5. Camarano, A.A.; Kanso, S.; Mello J.L.; Pasinato M.T. (2004). Famílias: espaço de compartilhamento de recursos e vulnerabilidades. Capítulo 5. In: Camarano AA, organizadora. Os novos idosos brasileiros muito além dos 60? Rio de Janeiro: IPEA; 137-167. Recuperado em 04 de Novembro de 2020, de: https://www.scielo.br/scielo.php?script=sci_artt ext\&amp;pid=S1413-81232019000401309.

6. Silva IT, Junior EPP, Vilela ABA. Autopercepção de saúde de idosos que vivem em estado de corresidência. Rev. Bras. Geriatr. Gerontol. 2014; 17(2): 275-287.

7. Santos $\mathrm{KL}$, Eulálio $\mathrm{MC}$, Júnior $\mathrm{EGS}$, Pessoa MCB, Melo RLP. Idosos na atenção primária à saúde: qualidade de vida e características associadas. Estud. psicol. 2019; 36: e180107.

8. Sampaio TSO, Sampaio LS, Vilela ABA. Conteúdos e estrutura representacional sobre família para idosos em corresidência. Ciência \& Saúde Coletiva. 2019; 24(4):1309-1316.

9. BRASIL. Ministério da Saúde. Secretaria de Atenção à Saúde. Departamento de Atenção Básica. Caderno de Atenção Básica n. 19 Envelhecimento e Saúde da pessoa idosa. Brasília: Ministério da Saúde, 2007

10. Veras R, Dutra S. Perfil do idoso brasileiro: questionário boas. UERJ/UnATI. 2008.

11. Meira SS, Vilela ABA, Casotti CA, Nascimento JC, Andrade CB. Idosos em estado de corresidência em um município do interior da Bahia. Mundo da Saúde. 2015; 39(2): 201-209.

12. Junior EPP, Silva IT, Vilela ABA, Casotti $C A$ et al. Dependência funcional e fatores associados em idosos corresidentes. Cad. Saúde Colet. 2016; 24 (4): 404-412

13. Souza AS, Vilela ABA, Sampaio LS, Soares GM, Silva JOL, Rocha PR, et al. Satisfação e estilo de vida de idosos corresidentes. International Journal of Development Research. 2020; 10 (07): 37459-37463.

14. Cruz MS, Araújo JA, Paixão AN. Family structure and its impacts on the restrictions of self-perception of elderly health levels in Brazil. 
Ciência \& Saúde Coletiva. 2018; 23(8): 27512762.

\section{Endereço para Correspondência}

Caroligia Pereira de Souza

Rua Claudionor de Oliveira, número 210, bairro

Candeias- Vitória da Conquista- BA

E-mail: carol.ligia21@gmail.com

Recebido em 02/04/2021

Aprovado em 11/06/2021

Publicado em 27/09/2021 